\title{
Red yeast rice induces less muscle fatigue symptom than simvastatin in dyslipidemic patients: a single center randomized pilot trial
}

Yangjing Xue ${ }^{1}$, Luyuan Tao ${ }^{1}$, Shaoze Wu' ${ }^{1}$ Guoqiang Wang ${ }^{1}$, Lu Qian¹, Jiwu Li', Lianming Liao², Jifei Tang ${ }^{\text {* }}$ and Kangting $\mathrm{Ji}^{1^{*}}$ (D)

\begin{abstract}
Background: About 10-15\% patients who take statins experience skeletal muscle problems. Red yeast rice has a good safety profile could provide a compromise therapeutic strategy. Therefore, the aim of this study was to evaluate the effects of red yeast rice, when compared to simvastatin, on the muscle fatigue symptom and the serum lipid level in dyslipidemic patients with low to moderate cardiovascular risk.

Methods: A total of 60 dyslipidemic patients with low to moderate cardiovascular risk were recruited and randomly assigned to receive either simvastatin $(n=33)$ or red yeast rice $(n=27)$ for 4 weeks. The muscle fatigue score, the physical activity, the serum lipid profile and the safety profile were then evaluated.

Results: At the end of study, the fatigue score was significantly increased in patients treated with simvastatin, whereas no significant change was observed in patients receiving red yeast rice. In addition, the physical activity level was significantly decreased in patients from simvastatin group when compared to those from red yeast rice group. Similar lipid-lowering effects were observed in two groups. The safety profile was not affected after the treatments.
\end{abstract}

Conclusions: Among dyslipidemic patients with low to moderate cardiovascular risk, red yeast rice induced less fatigue side effect and exerted comparable lipid-lowering effects when compared to simvastatin in this pilot primary prevention study.

Trial registration: NCT01686451.

Keywords: Muscle fatigue symptom, Physical activity, Red yeast rice, Simvastatin, Dyslipidemia, Lipid-lowering effects

\section{Background}

Statins have been shown to be beneficial for both primary and secondary cardiovascular (CV) prevention [1-5]. However, despite the efficacies of these agents in terms of lowering lipid levels, the rate of CV events, and, in some samples, mortality, some patients are unable to tolerate the adverse effects [6]. Unfortunately, failure to adhere to statin therapy can result in adverse CV outcomes $[7,8]$.

\footnotetext{
* Correspondence: jiftang@126.com; jikt@wzmc.edu.cn

${ }^{1}$ Department of Cardiology, The Second Affiliated Hospital and Yuying

Children's Hospital of Wenzhou Medical University, Xueyuanxi Road, No 109,

Wenzhou, Zhejiang 325000, China

Full list of author information is available at the end of the article
}

Increased fatigue has been recognized as one of the adverse effects of statins [9-13]. In a recent large scale randomized controlled trial, the majority of the patients were reported experiencing fatigue [13, 14]. Red yeast rice, which is a traditional dietary seasoning from the MONASCUS purpureus mold that contains lovastatin (Monacolin K) and other active ingredients, has been shown to exert lipid-lowering effects and CV benefits in both primary and secondary CV prevention studies [15-17]. Additionally, Red yeast rice is associated with few adverse events [15-17]. Unexpectedly, red yeast rice has been reported to have antifatigue effects [18]. 
In the present study, we compared the efficacy and fatigue-causing effects of red yeast rice and simvastatin in patients with dyslipidemia and moderate to low $\mathrm{CV}$ risk.

\section{Methods \\ Participants}

Patients with low-density lipoprotein-cholesterol (LDL-C) levels between 3 and $5 \mathrm{mmol} / \mathrm{L}(115-190 \mathrm{mg} / \mathrm{dL})$ were enrolled. Patients were excluded if they were at a very high to high risk of fatal CV disease within 10 years based on the risk estimation system described in ESC/ESA guidelines (2011) of dyslipidemia management, [19] or they had symptomatic atherosclerotic disease (including coronary artery disease, peripheral arterial disease, and cerebrovascular disease), kidney failure or insufficiency, diabetes, a systematic coronary risk estimation (SCORE) value $\geq 5 \%$, if they were currently using of any lipid-lowering medications or other medication, such as cyclosporin, erythromycin, clarithromycin, nefazodone, or any "azole" antifungal, (including fluconazole, itraconazole, ketoconazole, mibefradil, or protease inhibitors), or if they had conditions including active liver disease or unexplained persistently elevated transaminase levels, cancer, human immunodeficiency virus infection, a medical or psychiatric condition that prevented full study participation or follow-up (e.g., active psychosis), major surgery or hospitalization in the 3 months prior to study entry, if they were a female of childbearing potential, or if they were currently participating in another clinical trial.

The study was approved by the institutional review board, and all patients provided written informed consent.

\section{Randomization and masking}

This trial was designed as a single-center, parallel-group study that took place at the medical clinic of the Second Affiliated Hospital of Wenzhou Medical University, China. According to the principle of the minimum distribution imbalance index, [20] the patients were randomly assigned to receive either red yeast rice (4 pills each containing $300 \mathrm{mg}$, hence in total $1200 \mathrm{mg}$ daily) or simvastatin (0.5 pills each containing $40 \mathrm{mg}$, hence in total $20 \mathrm{mg}$ daily) for 4 weeks. Patients were reminded weekly by telephone to take the medication on schedule and were asked to revisit at $28 \pm 1$ days (week 4).

\section{Study procedures}

Fasting blood samples were collected at week 0 (randomization) and 4 (at end of study) for lipid profile analyses and clinical chemistry (including serum lipid concentrations, alanine transaminase (ALT), aspartate transaminase (AST), creatine phosphate kinase $(\mathrm{CPK})$ and serum creatinine $(\mathrm{Cr}))$. All clinical laboratory analyses were performed in the hospital's central laboratory.

\section{Outcome measures}

The primary endpoint was the fatigue score at the end of the study. Physical activity levels were also estimated. The baseline value was defined as the mean of the measurements obtained 1 week before randomization and on the day of randomization. The end-point value was defined as the measurement acquired after 4 weeks of treatment.

We estimated different aspects of fatigue levels with psychological and physical questionnaires. The fatigue scores were assessed with a fatigue questionnaire [21]. Changes in physical activity levels as evaluated with a short version of the international physical activity questionnaire [22] was also recorded. Because of drugs' preparation issue, the measurement of the questionnaire was conducted in a single-blinded method that the physician did not know the treatment that patients received.

The predefined secondary efficacy endpoints included the percentage changes from baseline to the study endpoint in lipid parameters (triglyceride (TG), total cholesterol (TC), high-density lipoprotein-cholesterol (HDL-C), and LDL-C levels).

Safety was assessed by recording the prevalence and severity of adverse events and abnormal laboratory data. The patients who reported adverse events were also included.

Compliance was assessed at each visit by counting the number of returned tablets.

\section{Statistical analyses}

All analyses were performed based on the intention-totreat principle using data from all of the randomized patients. There were no treatment crossovers in the 4week study period. Depending on the distributions, the continuous data are presented as medians (25th to 75 th percentiles) or as the mean \pm SD. The categorical data are presented as counts or proportions. The differences between groups were assessed with $\chi^{2}$ tests or Fisher's exact tests for the categorical data and with the nonparametric Wilcoxon rank-sum test or Student's $t$ test for the continuous data. A 2-tailed value of $P<0.05$ was considered to indicate statistical significance. All statistical analyses were performed using SPSS 17.0 software for Windows (SPSS for Windows version 17.0, Chicago, IL, USA).

\section{Results}

\section{Patient characteristics}

From August 10, 2012 to September 15, 2013, 243 patients were screened, and 60 patients who met the inclusion criteria were enrolled and randomly assigned to either the simvastatin (33 patients) or red yeast rice (27 patients) group. The flow of participants through the study is presented in Fig. 1. Table 1 shows the 


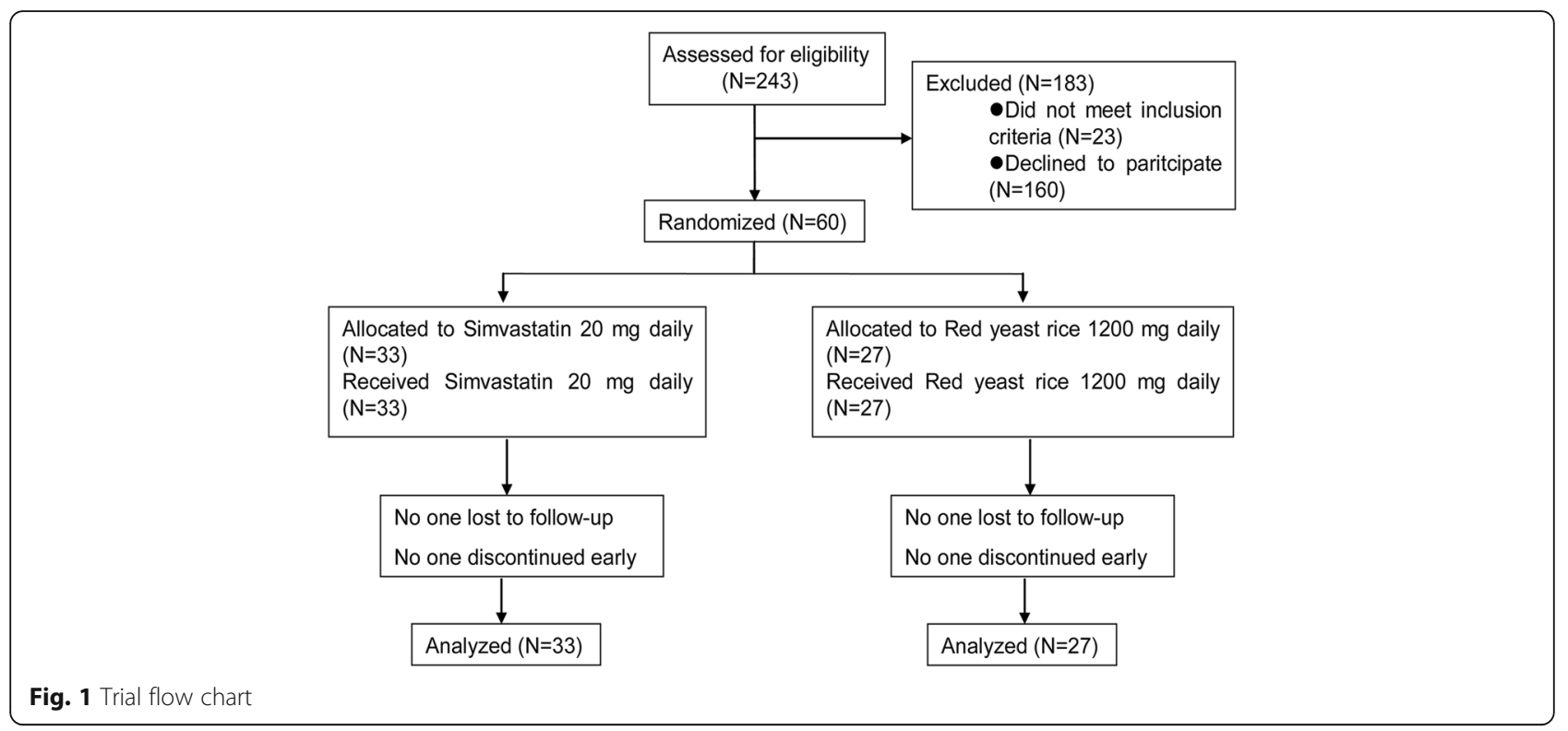

Table 1 Baseline characteristics of the patients ${ }^{a}$

\begin{tabular}{|c|c|c|c|}
\hline Characteristic & $\begin{array}{l}\text { Simvastatin } \\
(n=33)\end{array}$ & $\begin{array}{l}\text { Zuezhikang } \\
(n=27)\end{array}$ & $P$ value \\
\hline Female sex, no. (\%) & $15(45.5)$ & $13(48.2)$ & 0.835 \\
\hline Age, yr & $46.0 \pm 7.0$ & $47.0 \pm 5.8$ & 0.614 \\
\hline Current cigarette smoker, no. (\%) & $3(9.1)$ & $2(7.4)$ & 1.000 \\
\hline Arterial hypertension, no. (\%) & $26(78.8)$ & $16(59.3)$ & 0.101 \\
\hline \multicolumn{4}{|l|}{ Arterial blood pressure, $\mathrm{mmHg}$} \\
\hline Systolic & $148 \pm 19$ & $148 \pm 17$ & 0.971 \\
\hline Diastolic & $84 \pm 7$ & $83 \pm 9$ & 0.564 \\
\hline $\mathrm{FBG}, \mathrm{mmol} / \mathrm{L}$ & $5.10 \pm 0.36$ & $5.33 \pm 0.52$ & 0.048 \\
\hline $\mathrm{TC}, \mathrm{mmol} / \mathrm{L}$ & $5.91 \pm 0.71$ & $5.82 \pm 0.73$ & 0.488 \\
\hline $\mathrm{TG}, \mathrm{mmol} / \mathrm{L}$ & $1.76 \pm 1.00$ & $1.61 \pm 0.65$ & 0.633 \\
\hline $\mathrm{LDL}-\mathrm{C}, \mathrm{mmol} / \mathrm{L}$ & $3.72 \pm 0.48$ & $3.74 \pm 0.55$ & 0.509 \\
\hline $\mathrm{HDL}-\mathrm{C}, \mathrm{mmol} / \mathrm{L}$ & $1.27 \pm 0.25$ & $1.23 \pm 0.25$ & 0.906 \\
\hline$A L T, U / L$ & $28.6 \pm 10.6$ & $30.3 \pm 13.3$ & 0.570 \\
\hline AST, U/L & $24.1 \pm 7.0$ & $25.6 \pm 7.5$ & 0.425 \\
\hline $\mathrm{CPK}, \mathrm{U} / \mathrm{L}$ & $80.80 \pm 24.90$ & $91.7 \pm 23.7$ & 0.092 \\
\hline $\mathrm{Cr}, \mu \mathrm{mol} / \mathrm{L}$ & $72.6 \pm 14.4$ & $75.6 \pm 17.2$ & 0.463 \\
\hline Fatigue score & $19.6 \pm 2.6$ & $19.3 \pm 1.9$ & 0.592 \\
\hline Physical activity level & & & 0.870 \\
\hline Low, no. (\%) & $14(42.4)$ & $3(11.1)$ & \\
\hline Moderate, no. (\%) & $10(30.3)$ & $12(44.4)$ & \\
\hline High, no. (\%) & $9(27.3)$ & $12(44.4)$ & \\
\hline
\end{tabular}

${ }^{a}$ The plus-minus values are the means \pm the SDs. The percentages do not sum to 100 due to rounding. ALT alanine transaminase, AST aspartate transaminase, $C P K$ creatine phosphate kinase, $\mathrm{Cr}$ creatinine, $F B G$ fasting blood glucose, $H D L$ highdensity lipoprotein-cholesterol, $L D L$ low-density lipoprotein-cholesterol, $T C$ total cholesterol. TG triglyceride baseline characteristics of the patients, which were well balanced between the two treatment groups.

\section{Efficacy results}

Medication adherence was assessed by pill counting. All patients completed the self-rated fatigue assessment scale and the international physical activity questionnaire (short version) at the time of randomization and at week 4 . The baseline values were comparable between the simvastatin and red yeast rice groups (Table 1). At week 4 , the fatigue scores were significantly increased in the simvastatin group $(P<.001$ vs. baseline) and were significantly greater than those of the red yeast rice group $(P<.01$; Fig. 2$)$. The fatigue scores did not change in the red yeast rice group ( $P=.16$ vs. baseline; Fig. 2$)$. Similarly, the physical activity levels, which were significantly reduced in the simvastatin group $(P<.001$ vs. baseline), remained unchanged in the red yeast rice group $(P=.19$ vs. baseline; Table 2$)$ and were significantly lower in the simvastatin group than in the red yeast rice group $(P<.001)$ at week 4 (Table 3$)$.

Before lipid-lowering treatment, there is no significant difference neither between the two groups of baseline level of TC(5.91 \pm 0.71 vs. $5.82 \pm 0.73 \mathrm{mmol} / \mathrm{L}$ for $\mathrm{sim}$ vastatin and red yeast rice groups respectively, ns), nor between the two groups of baseline level of LDL$\mathrm{C}(3.72 \pm 0.48 \mathrm{vs}$. $3.74 \pm 0.55 \mathrm{mmol} / \mathrm{L}$ for simvastatin and red yeast rice groups respectively, ns). The administration of both simvastatin ( $20 \mathrm{mg}$ daily) or red yeast rice (1200 mg daily) resulted in significant reductions in TC $(-19.6 \%$ vs. $-18.5 \%$ of baseline level for simvastatin and red yeast rice groups respectively, $P<.001$ vs. baseline for both) and LDL-C (-30.9\% vs.-33.4\% of baseline level for simvastatin and red yeast rice groups respectively, 


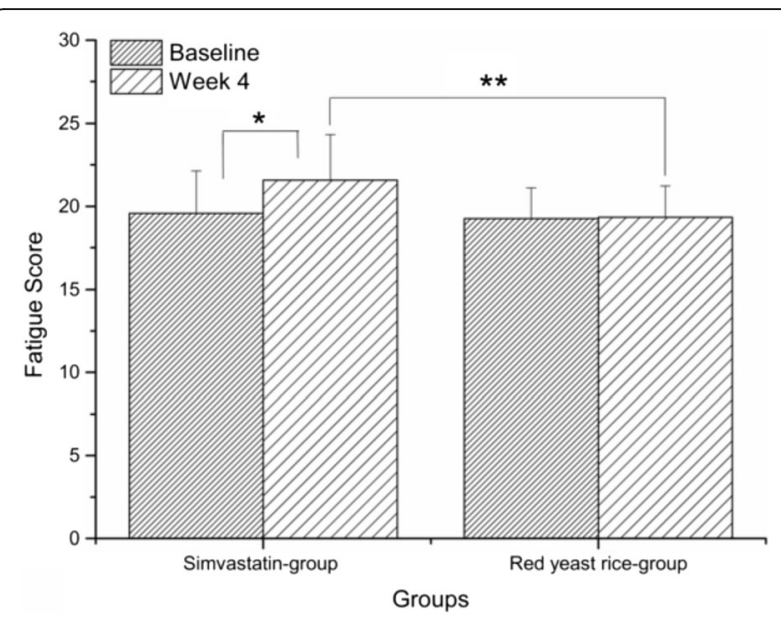

Fig. 2 Comparison of the fatigue scores of the simvastatin and red yeast rice groups (mean $\pm \mathrm{SD}$ ). ${ }^{*} P<.001 \mathrm{vs}$. baseline in the simvastatin group. ${ }^{*} P<.01$ between the simvastatin and red yeast rice groups at week 4

$P<.001$ vs. baseline for both) after 4 weeks of treatment (Fig. 3a-b) that were not significantly different between the two groups $(P=0.84$ for the comparison of the percentage drop in the TC level and $P=0.64$ for the comparison of the percentage drop in the LDL-C level; Fig. 3c). The improvements in TG and HDL-C concentrations were not significant in either treatment group (Fig. 3a-b).

\section{Safety results}

No significant increases of in the concentrations of ALT, AST, Cr, or CPK from baseline were observed in either arm (Fig. 4). No patient reported any adverse events.

\section{Discussion}

Dealing with dyslipidemia should always be considered to be an essential and integral part of cardiovascular disease (CVD) prevention, and the tailoring of interventions to the baseline level of CVD risk is rational $[19,23]$. In this trial, we compared the safeties and efficacies of simvastatin (20 mg daily) and red yeast rice (1200 mg daily) for patients with dyslipidemia and a 10 -year risk of fatal CVD between $1 \%$ and $5 \%$ as evaluated with the SCORE system [19]. The findings indicated that the two treatments were comparable in terms of the decreases in LDL-C and TC levels. However, unlike the patients in the simvastatin group, the patients in the red yeast rice group did not experience fatigue or decreased physical activity.

Statins are generally well tolerated and rarely cause serious adverse events [1-5]. In the Cholesterol Treatment Trialist's (CTT) meta-analyses that incorporated 26 randomized controlled trials, no increases in the risks for any non-CV cause of death and a small non-significant increase in rhabdomyolysis were observed in patients who were receiving statins [3]. Subsequent CTT studies and other meta-analyses that have addressed the issues of primary CV prevention have reported similar conclusions $[1,2,4,5]$.

However, statin-related fatigue has anecdotally been reported and confirmed by several studies, [9-14] including one randomized controlled trial [13] that enrolled 1016 participants (692 men and 324 women) who did not have heart disease or diabetes. The participants were randomly assigned to receive one of two statins (simvastatin or pravastatin) or placebo daily for six months. After six months, the participants taking the statins exhibited greater increases in overall fatigue than those who were taking the placebo. This effect was particularly pronounced in the women. The underlying mechanisms of statin-associated fatigue remain unclear but might be categorized as a manifestation of myopathy due to mitochondrial dysfunction [24-27]. Doctors should take these issues into account when considering the prescription of statins.

Although the potential benefits of prescribing statins to a patient who is at high risk of $\mathrm{CV}$ problems might outweigh the risks of side effects, such as increased levels of fatigue, the opposite might be true for a person who is at a low risk for CV problems. This balance should be decided on a patient-by-patient basis by the doctor and the patient. An alternative approach is the selection of medications other than statins. In the present study, we compared the safeties, specifically in terms of fatigue, and efficacies of simvastatin and red yeast rice in patients with dyslipidemia and moderate to low CVD risks. Red yeast rice is a Chinese herbal medication that has been approved by the China Food and Drug Administration for dyslipidemia. Red yeast rice contains a family of monacolin-related substances, one of which is a naturally occurring lovastatin. In a large-scale randomized, placebo-controlled trial, called the China Coronary

Table 2 Comparison of the physical activity levels at baseline and at week 4

\begin{tabular}{|c|c|c|c|c|c|c|}
\hline & \multicolumn{2}{|c|}{ Simvastatin $(n=33)$} & \multirow{2}{*}{$\begin{array}{l}P \\
\text { value }\end{array}$} & \multicolumn{2}{|c|}{ Xuezhikang $(n=27)$} & \multirow{2}{*}{$\begin{array}{l}P \\
\text { value }\end{array}$} \\
\hline & Baseline & Week 4 & & Baseline & Week 4 & \\
\hline Physical activity level & & & $<.001$ & & & 0.19 \\
\hline Low, No. (\%) & $14(42.4)$ & $25(75.8)$ & & $3(11.1)$ & $3(11.1)$ & \\
\hline Moderate, No. (\%) & $10(30.3)$ & $8(24.2)$ & & $12(44.4)$ & $15(55.6)$ & \\
\hline High, No. (\%) & $9(27.3)$ & $0(0.0)$ & & $12(44.4)$ & $9(33.3)$ & \\
\hline
\end{tabular}


Table 3 Physical activity levels of two treatment groups at week 4

\begin{tabular}{llll}
\hline & $\begin{array}{l}\text { Simvastatin } \\
(n=33)\end{array}$ & $\begin{array}{l}\text { Red yeast rice } \\
(n=27)\end{array}$ & $P$ value \\
\hline Physical activity level & & & $<.001$ \\
Low, No. (\%) & $25(75.8)$ & $3(11.1)$ & \\
Moderate, No. (\%) & $8(24.2)$ & $15(55.6)$ & \\
High, No. (\%) & $0(0.0)$ & $9(33.3)$ & \\
\hline
\end{tabular}

Secondary Prevention Study, 4870 patients with prior myocardial infarctions and baseline cholesterol levels between $170 \mathrm{mg} / \mathrm{dL}-250 \mathrm{mg} / \mathrm{dL}$ (4.40-6.47 mmol/L) exhibited significantly reduced rates of $\mathrm{CV}$ events following red yeast rice treatment [16]. In a meta-analysis involving 9625 participants with primary hyperlipidemia, red yeast rice preparations appeared to be as effective as statins in lipid modification [15]. In these trials, [15] Xuezhikang (red yeast rice preparation) was used at dosage of $1.2 \mathrm{~g} /$ day (containing $10 \mathrm{mg}$ of lovastatin),
Zhibituo (red yeast rice preparation) at $3.15 \mathrm{~g} /$ day (containing $9 \mathrm{mg}$ of lovastatin), simvastatin at $10-20 \mathrm{mg} /$ day, pravastatin at $10 \mathrm{mg} /$ day, lovastatin at $20 \mathrm{mg} /$ day, atorvastatin $10 \mathrm{mg} /$ day, and fluvastatin $20 \mathrm{mg} /$ day. Moreover, in sharp contrast to simvastatin, we did not observe any significant effect of red yeast rice on fatigue scores or physical activity levels after 4 weeks of treatment. In the present study, we found that the lipid modification efficacies both of red yeast rice (at $1200 \mathrm{mg}$ daily) and simvastatin (at $20 \mathrm{mg}$ daily) were comparable. Although the beneficial changes were limited to the TC and LDL-C parameters, these changes were still highly important because the current guidelines recommend that LDL-C should be the primary target of therapy $[19,23]$.

There are some limitations to this research. First, the sample size was relatively small, and future studies with larger sample sizes are needed to validate our results. Secondly, we followed the participants for only 4 weeks; thus, the long-term outcomes remain to be determined.

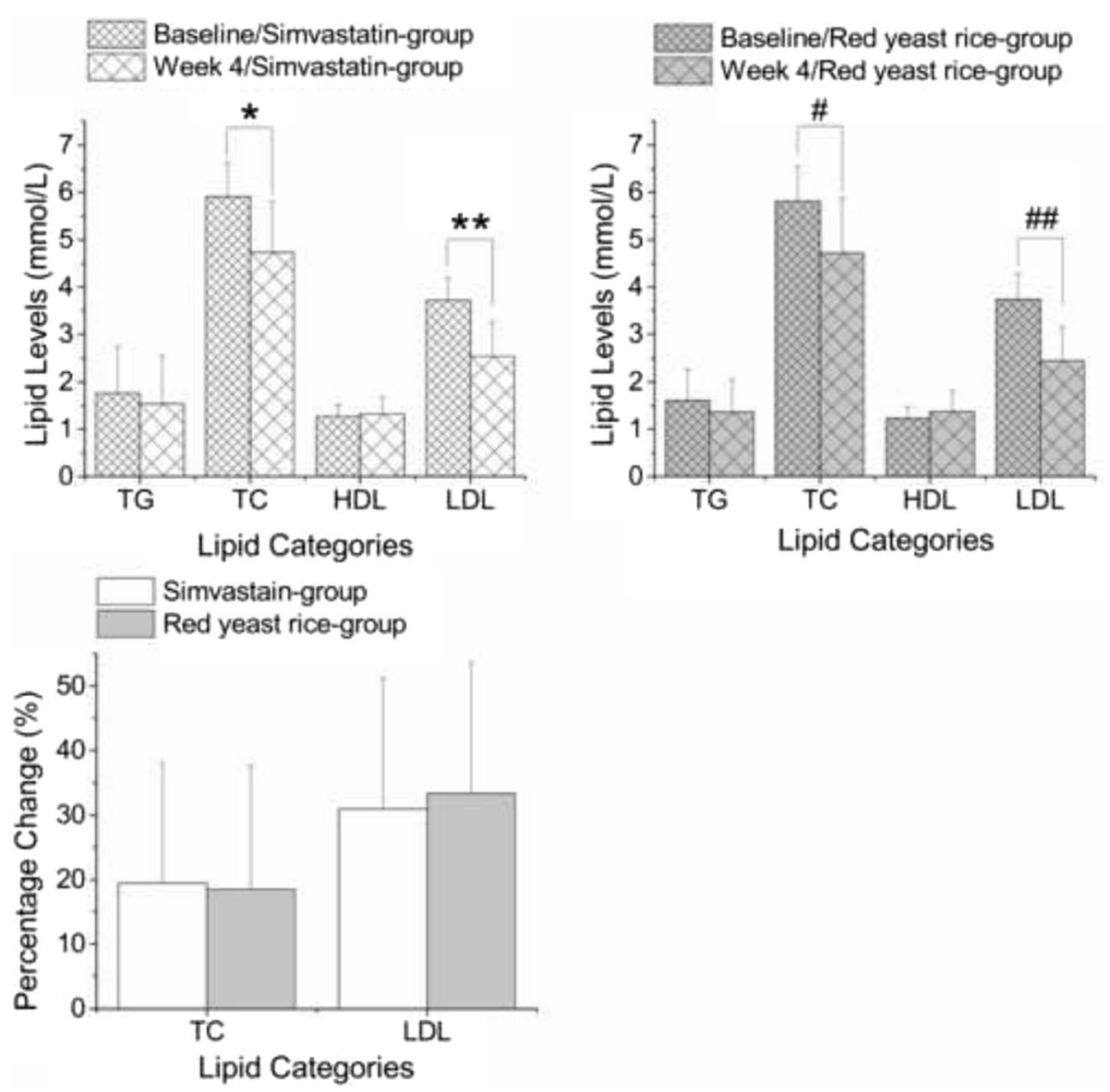

Fig. 3 Comparison of the lipid-lowering efficacies of simvastatin and red yeast rice; $\mathbf{a}$ \& $\mathbf{b}$ Comparison of lipid levels at week 4 and baseline in the simvastatin and red yeast rice groups (mean \pm SD). No significant changes in TG or HDL levels were observed in either arm; c Comparison of the percentage changes in the lipid (TC and LDL) levels from baseline to week 4 (mean \pm SD). The decrease in both the TC and LDL levels were comparable between the two groups.* TC levels, $P<.001$ vs. baseline in simvastatin group. ${ }^{*}$ LDL levels, $P<.001$ vs. baseline in simvastatin group. \# TC levels, $P<.001$ vs. baseline in red yeast rice group. \#\# LDL levels, $P<.001$ vs. baseline in red yeast rice group 

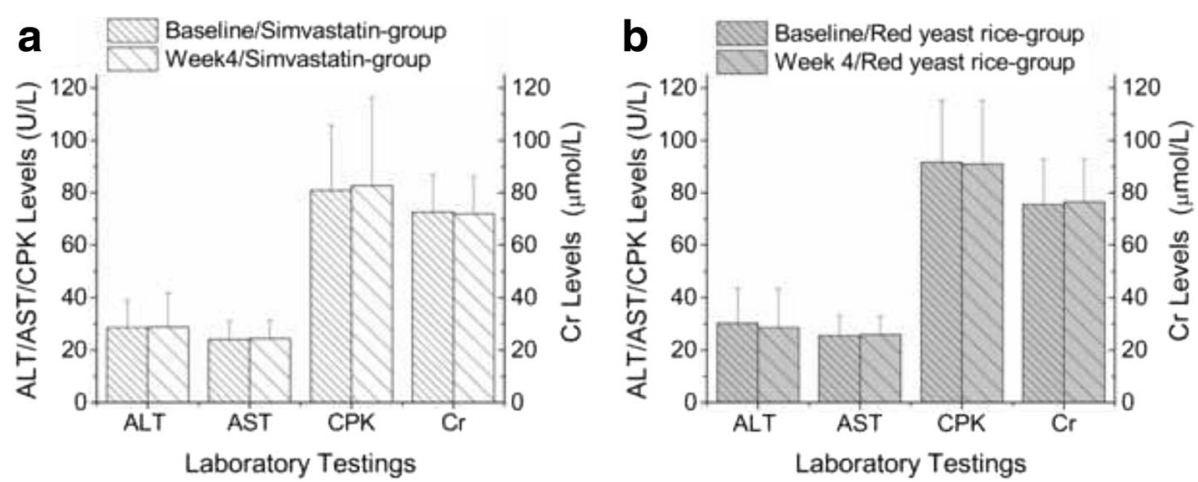

Fig. 4 Comparison of the safeties at week 4 and at baseline (mean \pm SD). No significant changes in ALT, AST, CPK or Cr levels were observed in either group

Thirdly, two possibilities might confound the effects of simvastatin and red yeast rice on fatigue score and physical activity level reported by the present pilot study: 1) small sample effects and potential baseline disparities in fatigue score, physical activity level, and hypertension; and 2) population characteristics that might place them at higher risk, e.g. Asian ethnicity coupled with a high fraction of hypertension.

\section{Conclusions}

Red yeast rice had similar lipid lowering properties to simvastatin in this small, short-term primary prevention trial, and may cause less fatigue. Further study of red yeast rice's long-term safety and efficacy in this patient population is warranted.

\section{Abbreviations \\ ALT: Alanine transaminase; AST: Aspartate transaminase; CPK: Creatine phosphate kinase; Cr: Serum creatinine; CTT: Cholesterol treatment trialist's; CV: Cardiovascular; CVD: Cardiovascular disease; HDL-C: High-density lipoprotein-cholesterol; LDL-C: Low-density lipoprotein-cholesterol; SCORE: Systematic coronary risk estimation; TC: Total cholesterol; TG: Triglyceride}

\section{Acknowledgements}

We acknowledge that the Second Affiliated Hospital and Yuying Children's Hospital of Wenzhou Medical University for supporting the work of our study.

\section{Funding}

Not applicable.

\section{Availability of data and materials}

The datasets generated during and/or analysed during the current study are available in the ClinicalTrials.gov repository, https://clinicaltrials.gov/ct2/show/ NCT01686451?term $=$ NCT01686451\&rank=1.

\section{Authors' contributions}

KTJ and JFT analyzed and interpreted the patient data. YJX was a major contributor in writing the manuscript. YJX, LYT, SZW, GQW, LQ, JWL, LML participated in data acquisition. All authors read and approved the final manuscript.

\section{Competing interests}

The authors declare that they have no competing interests.
Consent for publication

Not applicable.

\section{Ethics approval and consent to participate}

The study was approved by the institutional review board of the Second Hispital Affiliated to Wenzhou Medical University, and all patients provided written informed consent. The ethics approval number is 2012-27.

\section{Publisher's Note}

Springer Nature remains neutral with regard to jurisdictional claims in published maps and institutional affiliations.

\section{Author details}

'Department of Cardiology, The Second Affiliated Hospital and Yuying Children's Hospital of Wenzhou Medical University, Xueyuanxi Road, No 109, Wenzhou, Zhejiang 325000, China. ${ }^{2}$ Department of Oncology, Academy of Integrative Medicine, Fujian University of Traditional Chinese Medicine, Huatuo Road, No 1, Fuzhou, Fujian 350122, China.

Received: 30 July 2016 Accepted: 8 May 2017

Published online: 18 May 2017

\section{References}

1. Mills EJ, Rachlis B, Wu P, Devereaux PJ, Arora P, Perri D. Primary prevention of cardiovascular mortality and events with statin treatments: a network meta-analysis involving more than 65,000 patients. J Am Coll Cardiol. 2008; 52(22):1769-81.

2. Brugts JJ, Yetgin T, Hoeks SE, Gotto AM, Shepherd J, Knopp RH, Nakamura H, Ridker P, Van Domburg R, Deckers JW. The benefits of statin therapy in patients without cardiovascular disease: meta-analysis of randomized controlled trials. Eur Heart J. 2009:30:510-1.

3. Baigent C, Blackwell L, Emberson J, Holland LE, Reith C, Bhala N, Peto R, Barnes EH, Keech A, Simes J, et al. Efficacy and safety of more intensive lowering of LDL cholesterol: a meta-analysis of data from 170,000 participants in 26 randomised trials. Lancet. 2010;376(9753):1670-81.

4. Mihaylova B, Emberson J, Blackwell L, Keech A, Simes J, Barnes EH, Voysey M, Gray A, Collins R, Baigent C. The effects of lowering LDL cholesterol with statin therapy in people at low risk of vascular disease: meta-analysis of individual data from 27 randomised trials. Lancet. 2012;380(9841):581-90.

5. Taylor F, Huffman MD, Macedo AF, Moore TH, Burke M, Davey Smith G, Ward K, Ebrahim S. Statins for the primary prevention of cardiovascular disease. Cochrane Database Syst Rev. 2013;1:CD004816.

6. Cohen JD, Brinton EA, Ito MK, Jacobson TA. Understanding Statin use in America and gaps in patient Education (USAGE): an internet-based survey of 10,138 current and former statin users. J Clin Lipidol. 2012;6(3):208-15.

7. Ho PM, Bryson CL, Rumsfeld JS. Medication adherence: its importance in cardiovascular outcomes. Circulation. 2009;119(23):3028-35.

8. Puccetti L, Pasqui AL, Scarpini F, Cappellone R, Ghezzi A, Ceccatelli L, Auteri A. Statins discontinuation in compliant chronic users induces 
atherothrombotic profile despite baseline clinical setting and treatments. Int J Cardiol. 2011;153(3):328-9.

9. Bruckert E, Hayem G, Dejager S, Yau C, Begaud B. Mild to moderate muscular symptoms with high-dosage statin therapy in hyperlipidemic patients-the PRIMO study. Cardiovasc Drugs Ther. 2005;19(6):403-14.

10. McKenney JM, Davidson MH, Jacobson TA, Guyton JR. Final conclusions and recommendations of the National Lipid Association Statin Safety Assessment Task Force. Am J Cardiol. 2006;97(8A):89C-94C.

11. Cham S, Evans MA, Denenberg JO, Golomb BA. Statin-associated musclerelated adverse effects: a case series of 354 patients. Pharmacotherapy. 2010;30(6):541-53.

12. Joanisse D, Frenette J, Laflamme N, Dumont N, Gagne C, Couture P, Cote S, Piette V, Bergeron J. Statin withdrawal reduces muscle-related side effects and improves muscle function. Obes Facts. 2012;5:228.

13. Golomb BA, Evans MA, Dimsdale JE, White HL. Effects of statins on energy and fatigue with exertion: results from a randomized controlled trial. Arch Intern Med. 2012;172(15):1180-2.

14. Hoffman KB, Kraus C, Dimbil M, Golomb BA. A survey of the FDA's AERS database regarding muscle and tendon adverse events linked to the statin drug class. PLoS One. 2012;7:8.

15. Liu J, Zhang J, Shi Y, Grimsgaard S, Alraek T, Fonnebo V. Chinese red yeast rice (Monascus Purpureus) for primary hyperlipidemia: a meta-analysis of randomized controlled trials. Chin Med. 2006;1:4.

16. Lu Z, Kou W, Du B, Wu Y, Zhao S, Brusco OA, Morgan JM, Capuzzi DM, Li S. Effect of Xuezhikang, an extract from red yeast Chinese rice, on coronary events in a Chinese population with previous myocardial infarction. Am J Cardiol. 2008;101(12):1689-93.

17. Yang CW, Mousa SA. The effect of red yeast rice (Monascus Purpureus) in dyslipidemia and other disorders. Complement Ther Med. 2012;20(6):466-74.

18. Wang JJ, Shieh MJ, Kuo SL, Lee CL, Pan TM. Effect of red mold rice on antifatigue and exercise-related changes in lipid peroxidation in endurance exercise. Appl Microbiol Biotechnol. 2006;70(2):247-53.

19. Reiner Z, Catapano AL, De Backer G, Graham I, Taskinen MR, Wiklund O, Agewall S, Alegria E, Chapman MJ, Durrington P, et al. ESC/EAS guidelines for the management of dyslipidaemias: the task force for the management of dyslipidaemias of the European Society of Cardiology (ESC) and the European atherosclerosis society (EAS). Eur Heart J. 2011:32(14):1769-818.

20. Taves DR. Minimization: a new method of assigning patients to treatment and control groups. Clin Pharmacol Ther. 1974;15(5):443-53.

21. Michielsen HJ, De Vries J, Van Heck GL. Psychometric qualities of a brief selfrated fatigue measure: the fatigue assessment scale. J Psychosom Res. 2003; 54(4):345-52.

22. Craig CL, Marshall AL, Sjostrom M, Bauman AE, Booth ML, Ainsworth BE, Pratt M, Ekelund U, Yngve A, Sallis JF, et al. International physical activity questionnaire: 12-country reliability and validity. Med Sci Sports Exerc. 2003; 35(8):1381-95.

23. National Cholesterol Education Program (NCEP) Expert Panel on Detection Evaluation, and Treatment of High Blood Cholesterol in Adults (Adult Treatment Panel III). Third report of the National Cholesterol Education Program (NCEP) expert Panel on detection, evaluation, and treatment of high blood cholesterol in adults (adult treatment Panel III) final report. Circulation. 2002;106(25):3143-421.

24. Delliaux S, Steinberg JG, Lesavre N, Paganelli F, Oliver C, Jammes Y. Effect of long-term atorvastatin treatment on the electrophysiological and mechanical functions of muscle. Int J Clin Pharmacol Ther. 2006;44(6):251-61.

25. Dirks AJ, Jones KM. Statin-induced apoptosis and skeletal myopathy. Am J Physiol Cell Physiol. 2006;291 (6):C1208-12.

26. Mascitelli L, Pezzetta F. Physical activity in statin-treated patients. Int J Cardiol. 2009:134(1):136-7.

27. Kwak HB, Thalacker-Mercer A, Anderson EJ, Lin CT, Kane DA, Lee NS, Cortright RN, Bamman MM, Neufer PD. Simvastatin impairs ADP-stimulated respiration and increases mitochondrial oxidative stress in primary human skeletal myotubes. Free Radic Biol Med. 2012;52(1):198-207. 